\title{
Análise das alterações na curvatura corneana com implanteintra-estromal: estudo experimental em coelhos
}

\author{
Analysis of corneal curvature alteration following intrastromal corneal ring \\ implantation:experimental study in rabbits
}

\author{
Cinara Sakuma de Oliveira Godoy ${ }^{1}$ \\ Samia Ali Wahab² \\ Hamilton Moreira ${ }^{3}$ \\ Luciane Bug'man Moreira ${ }^{4}$ \\ Glaucio de Godoy ${ }^{5}$
}

Trabalho realizado no Hospital de Olhos do Paraná e no Hospital Universitário Evangélico de Curitiba, sendo apresentado como requisito parcial à obtenção do grau acadêmico de Doutor em Princípios da Cirurgia pela Universidade Federal do Paraná.

${ }^{1}$ Doutora em clínica cirúrgica pela Universidade Federal do Paraná - UFPR - Curitiba (PR) - Brasil.

${ }^{2}$ Doutora em clínica cirúrgica pela UFPR - Curitiba (PR) - Brasil.

${ }^{3}$ Doutor em Oftalmologia pela Universidade Federal de São Paulo - UNIFESP - São Paulo (SP) - Brasil. Professor Adjunto da UFPR - Curitiba (PR) - Brasil. Professor Assistente da Faculdade Evangélica do Paraná - Curitiba (PR) - Brasil.

${ }^{4}$ Doutora em Oftalmologia pela UNIFESP - São Paulo (SP) - Brasil.

${ }^{5}$ Médico oftalmologista do Hospital de Olhos do Paraná - Curitiba (PR) - Brasil.

Endereço para correspondência: Rua Coronel Dulcídio, 199 - Curitiba (PR) CEP 80420-170

E-mail: oliveiracinara@hotmail.com

Recebido para publicação em 10.04.2006

Última versão recebida em 26.09.2006

Aprovação em 18.10.2006

Nota Editorial: Depois de concluída a análise do artigo sob sigilo editorial e com a anuência do Dr. Bruno Castelo Branco sobre a divulgação de seu nome como revisor, agradecemos sua participação neste processo.

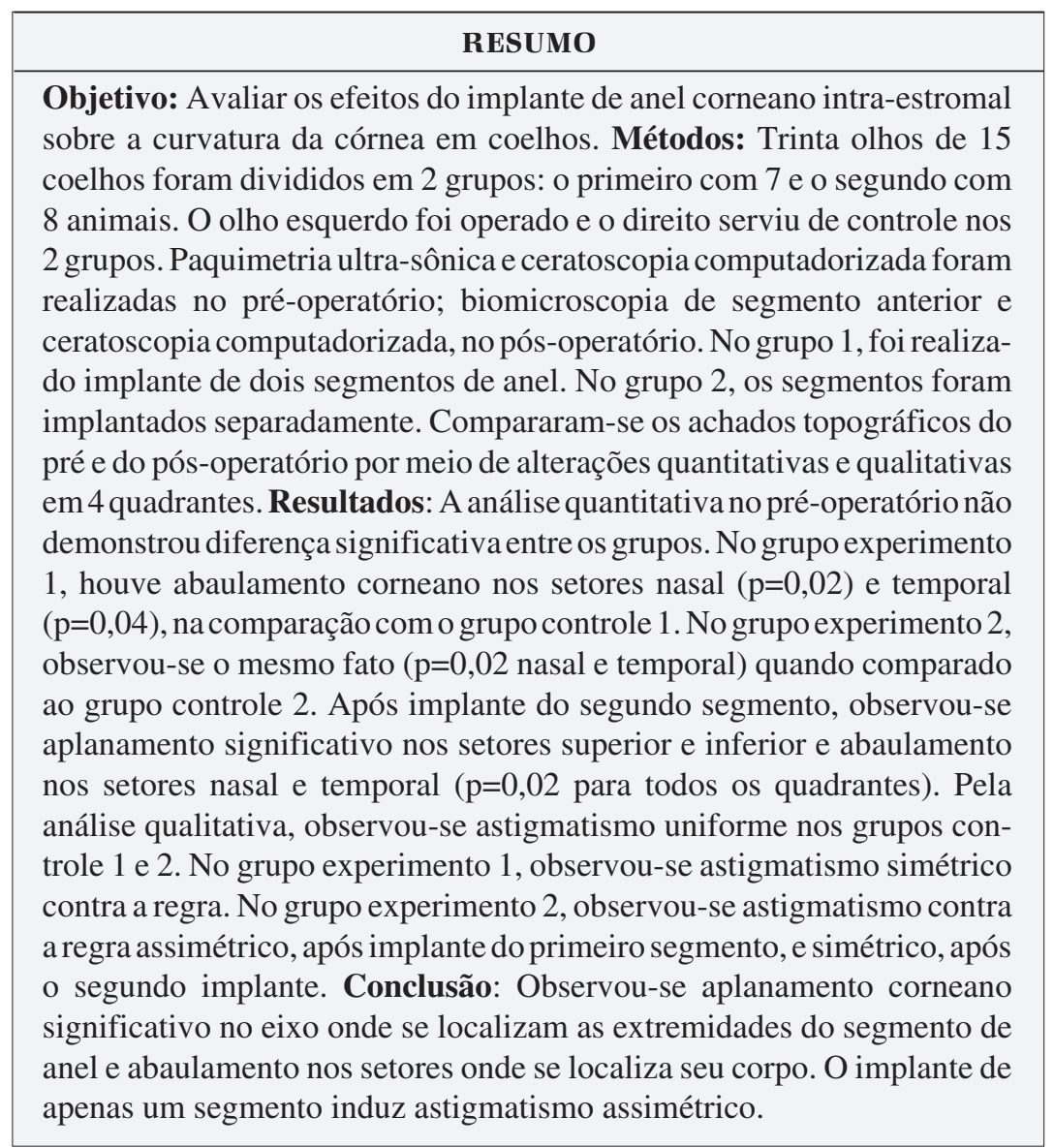

Descritores: Topografia da córnea; Implante de prótese; Estroma corneal/cirurgia; Astigmatismo; Coelhos

\section{INTRODUÇÃOO}

A córnea, por concentrar a maior parte do poder dióptrico do globo ocular e devido à sua fácil acessibilidade, tornou-se a principal estrutura anatômica modificada na cirurgia refrativa. Atualmente, os métodos mais empregados visam alterar sua curvatura por meio de incisões, subtração de tecido ou adição de material em sua periferia. Neste último caso, encontramse os anéis intra-estromais.

A forma de atuação destes anéis sobre a curvatura corneana tem sido motivo de estudos ao longo do tempo ${ }^{(1-6)}$. Pesquisas demonstram que 
quanto maior a espessura dos segmentos, maior o aplanamento obtido ${ }^{(3)}$. Desta forma, anéis inseridos nas regiões corneanas paracentral e periférica induziriam aplanamento corneano central. Tal efeito seria útil na correção de problemas como miopia e astigmatismo irregular ${ }^{(3)}$.

Em 1995, foram publicados os resultados da Fase I do FDA (Food and Drug Administration) dos estudos sobre o uso de anéis intra-estromais para correção de miopia. Foram avaliados 10 olhos não funcionais e se obteve aplanamento corneano médio de 2,5 dioptrias após 12 meses de acompanhamento $^{(4)}$. A configuração atual destes implantes, com dois segmentos simétricos, foi obtida após a segunda etapa destes estudos $^{(5)}$. Após a conclusão da terceira fase, o FDA aprovou, em 1999, a utilização de anéis corneanos intra-estromais na correção de miopia. Nesta fase, $99 \%$ dos pacientes submetidos a este procedimento atingiram acuidade visual não corrigida de 20/40 ou melhor após um ano de acompanhamento. Noventa e dois por cento dos pacientes apresentaram diferença entre a refração pós-operatória e a refração esperada no pré-operatório de uma dioptria. Estabilidade refracional foi atingida após três meses de cirurgia ${ }^{(6)}$.

Em 2000, foram realizados estudos que propuseram uso de anel intra-estromal para redução do astigmatismo, correção esférica e aumento da regularidade topográfica em pacientes com ceratocone ${ }^{(7)}$. Em 2001 foram publicados os resultados com o uso do INTACS para a correção do ceratocone após um ano de seguimento. Em seus estudos, a média da acuidade visual corrigida melhorou de 0,38 unidades de LogMAR para 0,22 unidades de $\log \mathrm{MAR}^{(8)}$.

A partir desse estudo, outras pesquisas demonstraram a eficácia deste implante na redução do abaulamento corneano e do astigmatismo irregular associados ${ }^{(9-14)}$. Estudos recentes têm mostrado que implante de segmentos assimétricos pode reduzir ainda mais a irregularidade corneana e melhorar de forma satisfatória a acuidade visual em pacientes com esta doença ${ }^{(14)}$.

Os estudos disponíveis até o momento, tanto para correção de miopia quanto para correção de ceratocone, em sua maior parte, foram conduzidos em seres humanos e baseados em achados clínicos. A literatura, entretanto, carece de estudos experimentais que demonstrem os efeitos do anel intra-estromal sobre a curvatura corneana, no que se refere ao grau de aplanamento obtido, à indução de astigmatismo, à utilização de segmentos assimétricos e ao posicionamento da incisão.

\section{OBJETIVO}

O objetivo deste trabalho é avaliar, por meio de estudo experimental, os efeitos do implante de anel corneano intraestromal sobre a curvatura corneana.

\section{MÉTODOS}

Foram estudados 30 olhos de 15 coelhos Oryctolagus cuniculus, da linhagem Nova Zelândia, albinos, machos, entre
2 e 4 meses de idade, pesando entre 1.500 a 2.400 gramas. Os animais foram divididos, de forma aleatória, em dois grupos com 7 animais no primeiro (Grupo 1) e 8 no segundo (Grupo 2). Em cada animal, o olho esquerdo foi submetido ao procedimento cirúrgico, e o olho direito, estudado como controle. Assim, cada grupo foi subdividido em grupos controle e experimento.

Todos os animais foram submetidos aos seguintes exames em ambos os olhos: paquimetria ultra-sônica, ceratoscopia computadorizada e biomicroscopia de segmento anterior.

Os animais do grupo experimento 1 foram submetidos ao implante de dois segmentos de anel corneano intra-estromal. Após 14 dias, foram submetidos aos exames de biomicroscopia de segmento anterior e ceratoscopia computadorizada e, em seguida, realizou-se eutanásia dos animais.

Os animais do grupo experimento 2 foram submetidos ao implante de um segmento de anel corneano intra-estromal no quadrante nasal da córnea. Após 14 dias, foram submetidos aos exames de biomicroscopia de segmento anterior e ceratoscopia computadorizada e, em seguida, realizou-se implante do segundo segmento de anel corneano. Após 28 dias da primeira cirurgia, estes animais foram novamente submetidos aos exames de biomicroscopia e ceratoscopia com posterior eutanásia.

Para os exames de ceratoscopia computadorizada, biomicroscopia e paquimetria ultra-sônica, foi realizada sedação com cloridrato de xylasina $\left(\right.$ Rompun $^{\mathrm{R}}$ ), na dose de $7 \mathrm{mg} / \mathrm{kg}$, com aplicação intramuscular. Nos procedimentos cirúrgicos, foi utilizado: cloridrato de ketamina $\left(\right.$ vetanarcol $^{\mathrm{R}}$ ), na dose de $40 \mathrm{mg}$ / $\mathrm{kg}$ intramuscular, cloridrato de xylasina $\left(\right.$ Rompun $\left.^{\mathrm{R}}\right)$ na dose de $7 \mathrm{mg} / \mathrm{kg}$ intramuscular e colírio de proparacaína a $0,5 \%$.

Ceratoscopia computadorizada foi realizada nos 30 olhos dos 15 coelhos, utilizando-se o topógrafo EyeSys Corneal Analysis System (Eye Sys Technologies, Dalas, EUA). Foram considerados os gráficos colorido e numérico para cada caso. Paquimetria ultra-sônica foi realizada nos 30 olhos, utilizandose o aparelho Sonomed $^{\circledR}$, no período pré-operatório, logo após o exame de ceratoscopia computadorizada. Foi realizada a $3 \mathrm{~mm}$ do centro da córnea, no setor superior às 12 horas.

Biomicroscopia de segmento anterior e documentação fotográfica foram realizadas após a cirurgia, com o animal sedado, antes de se realizar ceratoscopia computadorizada (Figura 1). Foi utilizada lâmpada de fenda com sistema fotográfico digital acoplado e ligado a um computador. Foram pesquisadas alterações biomicroscópicas como: descentração e extrusão dos segmentos de anel, hiperemia conjuntival, opacidade, infecção, erosão corneana e reação inflamatória na câmara anterior.

A técnica cirúrgica empregada foi a mesma descrita por Oliveira et al., em 2004, para o tratamento de ceratocone, no entanto, a incisão foi realizada sempre no setor superior, às 12 horas, atingindo $80 \%$ da espessura corneana segundo a paquimetria. Os segmentos de anel eram compostos de PMMA (polimetilmetacrilato), com espessura constante de 350 micra. Cada segmento possuía comprimento de 150 graus de arco e diâmetro de ápice de $5 \mathrm{~mm}$, apresentando uma secção triangular. No pós-operatório, foi utilizado colírio de associação de 


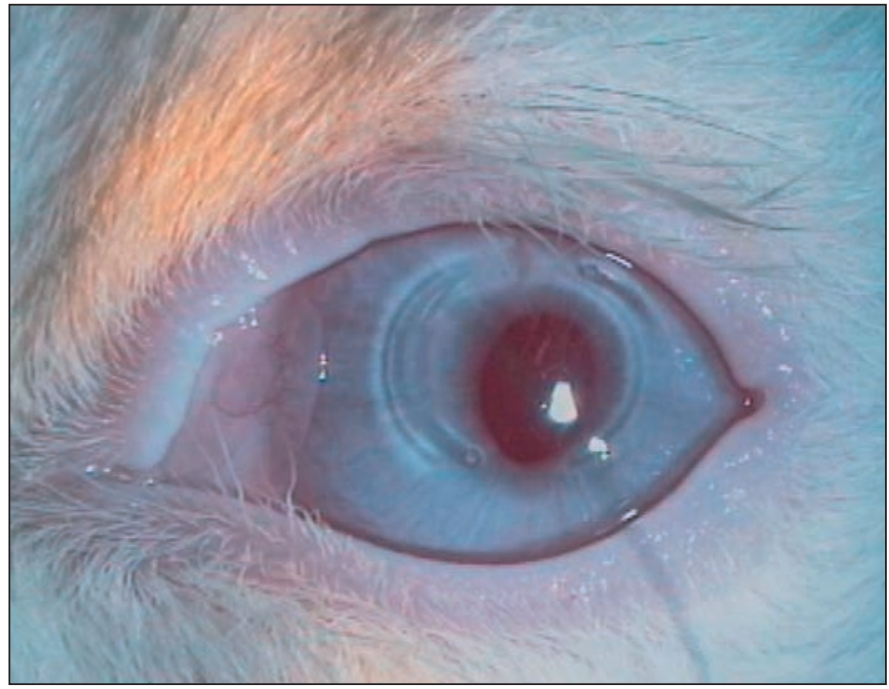

Figura 1 - Biomicroscopia de animal do grupo 1, com dois segmentos de anel, no $14^{\circ}$ pós-operatório

dexametasona $0,1 \%$ e tobramicina $0,3 \%$ quatro vezes ao dia na primeira semana e duas vezes ao dia por mais 7 dias.

Para a análise quantitativa das alterações topográficas corneanas, foram considerados os valores obtidos pelo gráfico numérico da ceratoscopia computadorizada. O mapa obtido foi dividido em quatro setores: superior, inferior, temporal e nasal. Em cada um deles, foram obtidos os seis valores ceratométricos mais centrais da curvatura corneana (em dioptrias) e calculada a média aritmética. Foi realizada uma análise intergrupos, ou seja, os valores obtidos em cada setor foram comparados entre os grupos, durante todo o período de estudo. Foi também realizada análise intragrupo, comparando-se os resultados pré e pós-operatório, no mesmo grupo de estudo.

A análise qualitativa da córnea foi realizada considerandose os resultados obtidos pelo mapa colorido mediante a classificação de Bogan et al. (1990) para o padrão de astigmatismo encontrado: uniforme, regular simétrico, regular assimétrico ou irregular.

Foi realizada pesagem de todos os animais com o objetivo de verificar-se a influência do crescimento corpóreo sobre a curvatura corneana. Os animais foram pesados antes da cirurgia, após 14 dias (Grupo 1) e após 14 e 28 dias (Grupo 2). Em uma primeira análise, comparou-se a homogeneidade entre os animais dos grupos 1 e 2 e o ganho de peso dos mesmos durante o estudo. Em uma segunda análise, os animais foram divididos, após a primeira pesagem, em dois grupos: grupo mais leve (menos de 2.000 gramas) e grupo mais pesado (mais de 2.000 gramas). Em seguida, foram calculadas as médias de peso e ceratometria dos dois grupos e comparadas entre si. Com isso, objetivou-se identificar as alterações ceratométricas associadas ao crescimento do animal e diferenciá-las daquelas relacionadas ao experimento.

As variáveis quantitativas foram representadas por média, desvio-padrão, valores mínimo e máximo. Para comparações pareadas envolvendo mais de dois momentos, considerou-se o teste não-paramétrico de Friedman. Para comparações pareadas envolvendo dois momentos, considerou-se o teste não-paramétrico de Wilcoxon. Nas avaliações com amostras independentes, considerou-se o teste não-paramétrico de Mann-Whitney. Valores de $\mathrm{p}<0,05$ indicaram significância estatística.

\section{RESULTADOS}

Inicialmente, o estudo incluiu 30 olhos de 15 coelhos. O primeiro animal submetido à cirurgia (pertencente ao grupo 1 C1) sofreu perfuração corneana durante a confecção da incisão e foi eliminado do estudo. No dia três, um animal do grupo 2 (C14) evoluiu com infecção corneana no local do implante de um dos segmentos de anel e também foi excluído do estudo. Após a segunda cirurgia, um animal do grupo 2 (C15) morreu por motivos desconhecidos e seus resultados também foram excluídos do estudo. Desta forma, permaneceram 12 animais, sendo 6 em cada grupo.

Exceto no primeiro animal operado, todas as cirurgias transcorreram sem intercorrências, em um tempo médio de 15 minutos. Nenhum animal reagiu durante a cirurgia ou sofreu complicações anestésicas.

Os achados biomicroscópicos mais freqüentemente encontrados no pós-operatório foram: descentração do anel (2 casos), hiperemia conjuntival (5 casos) e leve opacidade corneana (1 caso). Infecção corneana foi observada em apenas um animal (C14), que foi excluído do estudo. Não ocorreram extrusão do anel, erosão corneana ou reação inflamatória de câmara anterior.

A paquimetria ultra-sônica pré-operatória variou de 326 a 414 micra, com uma média de $367,33 \pm 28,32$ para o olho direito e $355,16 \pm 20,83$ para o olho esquerdo.

A média de pesos, no pré-operatório, nos animais do grupo 1 foi de 1.891,67 gramas e, após 14 dias, houve ganho médio significativo de 358 gramas. No grupo 2, a média de pesos antes da cirurgia foi de $1.791,67$ gramas e aumentou, de forma significativa, 400 gramas após 14 dias e 791,66 gramas após 28 dias. No grupo mais leve (menor que 2.000 gramas), a média de pesos foi de 1.591,66 gramas e a média de ceratometria foi de 50,79 dioptrias. No grupo mais pesado (maior ou igual a 2.000 gramas), o peso médio foi de 2.091 gramas e a média ceratométrica foi de 49,24 dioptrias. Testou-se a hipótese nula de que os resultados de ceratometria fossem iguais nos dois grupos definidos pelo peso, versus a hipótese alternativa de resultados diferentes. Observou-se que o grupo mais pesado apresentava valores ceratométricos significativamente mais baixos.

Avaliando-se os resultados em cada grupo individualmente (análise intragrupo), observa-se que, no grupo controle 1, após 14 dias, houve diminuição significativa das medidas ceratométricas nos setores superior, nasal e inferior. Apenas no setor temporal este valor não foi significativo.

No grupo controle 2, houve diminuição significativa das medidas ceratométricas em todos os setores da córnea. 
No grupo experimento 1 , houve diminuição significativa das medidas ceratométricas nos setores superior e inferior, enquanto nos setores nasal e temporal, houve aumento significativo destes valores (Tabela 1).

No grupo experimento 2 , houve diminuição significativa das medidas ceratométricas nos setores superior e inferior, enquanto nos setores nasal e temporal, houve aumento significativo destes valores (Tabela 2 e Gráfico). Nota-se aumento ceratométrico mais significativo no setor nasal, após 14 dias do procedimento, que diminuiu após 28 dias (Gráfico).

Os resultados foram comparados entre os grupos (análise intergrupos) nos três momentos: pré-operatório, 14 e 28 dias após a cirurgia.

\section{Pré-operatório}

Os valores obtidos no pré-operatório foram comparados entre todos os grupos, conforme indicado abaixo, não havendo diferença significativa, em nenhum setor da córnea, em nenhuma das comparações:

1. Grupo controle $1 \mathrm{X}$ Grupo controle 2

2. Grupo experimento $1 \mathrm{X}$ Grupo experimento 2

3. Grupo controle $1 \mathrm{X}$ Grupo experimento 1

4. Grupo controle 2 X Grupo experimento 2

\section{Pós-operatório - 14 dias}

1. Grupo controle $1 \mathrm{X}$ Grupo experimento 1

A tabela 3 demonstra que, após 14 dias de cirurgia, comparando-se os dois grupos, houve, no grupo experimento 1, diminuição dos valores ceratométricos nos setores superior e inferior da córnea e aumento significativo dos valores nos setores nasal e temporal.

2. Grupo controle $2 \mathrm{X}$ Grupo experimento 2

A tabela 4 demonstra que, após 14 dias de cirurgia, compa-

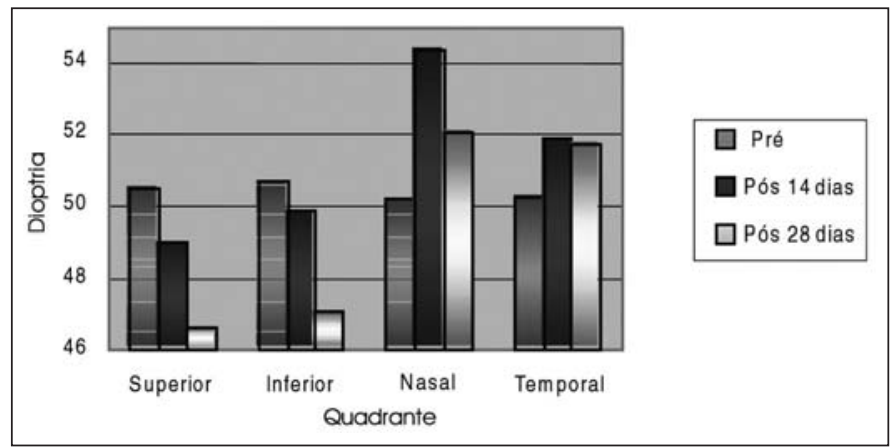

Gráfico - Comparação dos resultados pré e pós-operatórios (14 e 28 dias) dos valores ceratométricos no grupo experimento 2

\begin{tabular}{|c|c|c|c|c|c|c|c|c|}
\hline Variável & Grupo E1 & $\mathbf{N}$ & Média & Mediana & Mínimo & Máximo & Desvio-padrão & Valor de $p^{(*)}$ \\
\hline \multirow[t]{2}{*}{ Superior } & Pré & 6 & 49,62 & 49,66 & 47,47 & 51,71 & 1,43 & 0,0277 \\
\hline & Pós 14d & 6 & 47,40 & 46,67 & 44,86 & 50,71 & 2,15 & \\
\hline \multirow[t]{2}{*}{ Inferior } & Pré & 6 & 49,27 & 49,54 & 46,59 & 51,00 & 1,60 & 0,0464 \\
\hline & Pós 14d & 6 & 47,29 & 47,07 & 43,85 & 51,19 & 2,48 & \\
\hline \multirow[t]{2}{*}{ Nasal } & Pré & 6 & 49,13 & 49,13 & 46,82 & 51,29 & 1,60 & 0,0277 \\
\hline & Pós 14d & 6 & 51,53 & 51,54 & 48,00 & 55,12 & 2,45 & \\
\hline \multirow[t]{2}{*}{ Temporal } & Pré & 6 & 49,09 & 48,95 & 47,13 & 50,75 & 1,45 & 0,0277 \\
\hline & Pós 14d & 6 & 51,15 & 51,49 & 48,06 & 54,41 & 2,52 & \\
\hline
\end{tabular}

\begin{tabular}{|c|c|c|c|c|c|c|c|c|}
\hline Variável & Grupo E2 & $\mathbf{N}$ & Média & Mediana & Mínimo & Máximo & Desvio-padrão & Valor de $p^{(*)}$ \\
\hline \multirow[t]{3}{*}{ Superior } & Pré & 6 & 50,53 & 50,49 & 49,16 & 51,66 & 0,90 & 0,0025 \\
\hline & Pós 14d & 6 & 48,99 & 49,38 & 46,87 & 51,08 & 1,76 & \\
\hline & Pós 28d & 6 & 46,61 & 46,87 & 44,54 & 48,44 & 1,43 & \\
\hline \multirow[t]{3}{*}{ Inferior } & Pré & 6 & 50,68 & 50,69 & 49,67 & 51,92 & 0,73 & 0,0057 \\
\hline & Pós 14d & 6 & 49,85 & 49,68 & 47,99 & 51,61 & 1,52 & \\
\hline & Pós 28d & 6 & 47,08 & 46,97 & 45,50 & 48,42 & 1,05 & \\
\hline \multirow[t]{3}{*}{ Nasal } & Pré & 6 & 50,21 & 50,28 & 48,86 & 51,60 & 1,06 & 0,0302 \\
\hline & Pós 14d & 6 & 54,40 & 53,61 & 52,30 & 57,56 & 2,01 & \\
\hline & Pós 28d & 6 & 52,09 & 52,46 & 48,68 & 53,83 & 1,77 & \\
\hline \multirow[t]{3}{*}{ Temporal } & Pré & 6 & 50,22 & 50,24 & 49,30 & 51,59 & 0,80 & 0,0422 \\
\hline & Pós 14d & 6 & 51,86 & 51,68 & 50,12 & 54,20 & 1,39 & \\
\hline & Pós 28d & 6 & 51,77 & 52,40 & 48,74 & 53,44 & 1,75 & \\
\hline
\end{tabular}


rando-se os dois grupos, houve, no grupo experimento 2, discreta diminuição dos valores ceratométricos no setor superior da córnea (não significativa) e aumento significativo dos valores nos setores nasal e temporal.

\section{Pós-operatório - 28 dias}

1. Grupo controle 2 X Grupo experimento 2

A tabela 5 demonstra que, após 28 dias de cirurgia, comparando-se os dois grupos, houve, no grupo experimento 2, diminuição significativa dos valores ceratométricos no setor superior e inferior da córnea e aumento significativo dos valores nos setores nasal e temporal.

Por meio do mapa colorido da ceratoscopia computadorizada, analisou-se o padrão do astigmatismo encontrado durante o estudo. Nos animais do grupo controle 1 e do grupo controle 2, o padrão do astigmatismo não sofreu alterações significativas, comparando-se os mapas realizados nos dias 1, 14 e 28. Observou-se astigmatismo uniforme, definido como mapa videoceratográfico de padrão redondo ou oval, com diferença na escala de cores, ou entre o maior e o menor poder corneano, menor ou igual a uma dioptria. Nos animais do grupo experimento 1, o astigmatismo uniforme, observado nos exames pré-operatórios, foi alterado para astigmatismo regular simétrico contra a regra após a cirurgia. Em geral, nos animais do grupo experimento 2, o astigmatismo uniforme pré-operatório foi alterado para astigmatismo regular assimétrico contra a regra após o implante de apenas um segmento. Após o implante do segundo segmento, houve tendência a astigmatismo regular simétrico contra a regra (Figura 2).

\begin{tabular}{|c|c|c|c|c|c|c|c|c|}
\hline Variável & Grupo & $\mathbf{N}$ & Média & Mediana & Mínimo & Máximo & Desvio-padrão & Valor de $p^{(*)}$ \\
\hline \multirow[t]{2}{*}{ Superior } & Grupo C1 & 6 & 49,57 & 49,55 & 48,47 & 51,25 & 1,02 & 0,0747 \\
\hline & Grupo E1 & 6 & 47,40 & 46,67 & 44,86 & 50,71 & 2,15 & \\
\hline \multirow[t]{2}{*}{ Inferior } & Grupo C1 & 6 & 49,45 & 49,50 & 48,02 & 50,73 & 0,99 & 0,0747 \\
\hline & Grupo E1 & 6 & 47,29 & 47,07 & 43,85 & 51,19 & 2,48 & \\
\hline \multirow[t]{2}{*}{ Nasal } & Grupo C1 & 6 & 48,77 & 48,31 & 47,80 & 50,50 & 1,09 & 0,0277 \\
\hline & Grupo E1 & 6 & 51,53 & 51,54 & 48,00 & 55,12 & 2,45 & \\
\hline \multirow[t]{2}{*}{ Temporal } & Grupo C1 & 6 & 49,21 & 49,22 & 48,04 & 50,90 & 1,08 & 0,0464 \\
\hline & Grupo E1 & 6 & 51,15 & 51,49 & 48,06 & 54,41 & 2,52 & \\
\hline
\end{tabular}

\begin{tabular}{|c|c|c|c|c|c|c|c|c|}
\hline Variável & Grupo & $\mathbf{N}$ & Média & Mediana & Mínimo & Máximo & Desvio-padrão & Valor de $p^{(*)}$ \\
\hline \multirow[t]{2}{*}{ Superior } & Grupo C2 & 6 & 49,52 & 49,33 & 48,28 & 51,56 & 1,21 & 0,2489 \\
\hline & Grupo E2 & 6 & 48,99 & 49,38 & 46,87 & 51,08 & 1,76 & \\
\hline \multirow[t]{2}{*}{ Inferior } & Grupo C2 & 6 & 49,72 & 49,49 & 47,62 & 52,44 & 1,68 & 0,6002 \\
\hline & Grupo E2 & 6 & 49,85 & 49,68 & 47,99 & 51,61 & 1,52 & \\
\hline \multirow[t]{2}{*}{ Nasal } & Grupo C2 & 6 & 49,21 & 49,05 & 47,69 & 51,37 & 1,30 & 0,0277 \\
\hline & Grupo E2 & 6 & 54,40 & 53,61 & 52,30 & 57,56 & 2,01 & \\
\hline \multirow[t]{2}{*}{ Temporal } & Grupo C2 & 6 & 48,93 & 48,69 & 46,85 & 51,50 & 1,51 & 0,0277 \\
\hline & Grupo E2 & 6 & 51,86 & 51,68 & 50,12 & 54,20 & 1,39 & \\
\hline
\end{tabular}

\begin{tabular}{|c|c|c|c|c|c|c|c|c|}
\hline Variável & Grupo & $\mathbf{N}$ & Média & Mediana & Mínimo & Máximo & Desvio-padrão & Valor de $\mathbf{p}^{(*)}$ \\
\hline \multirow[t]{2}{*}{ Superior } & Grupo C2 & 6 & 48,47 & 48,25 & 46,63 & 51,28 & 1,56 & 0,0277 \\
\hline & Grupo E2 & 6 & 46,61 & 46,87 & 44,54 & 48,44 & 1,43 & \\
\hline \multirow[t]{2}{*}{ Inferior } & Grupo C2 & 6 & 48,57 & 48,52 & 47,02 & 51,52 & 1,63 & 0,0277 \\
\hline & Grupo E2 & 6 & 47,08 & 46,97 & 45,50 & 48,42 & 1,05 & \\
\hline \multirow[t]{2}{*}{ Nasal } & Grupo C2 & 6 & 48,27 & 48,20 & 46,52 & 50,99 & 1,56 & 0,0277 \\
\hline & Grupo E2 & 6 & 52,09 & 52,46 & 48,68 & 53,83 & 1,77 & \\
\hline \multirow[t]{2}{*}{ Temporal } & Grupo C2 & 6 & 48,02 & 47,56 & 46,60 & 50,90 & 1,56 & 0,0277 \\
\hline & Grupo E2 & 6 & 51,77 & 52,40 & 48,74 & 53,44 & 1,75 & \\
\hline
\end{tabular}




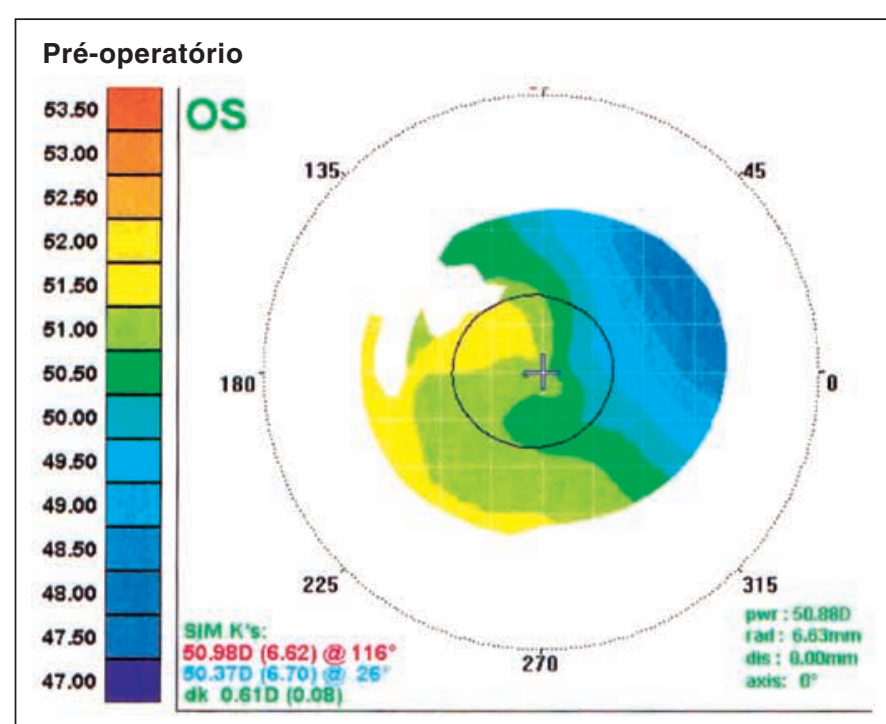

14 dias

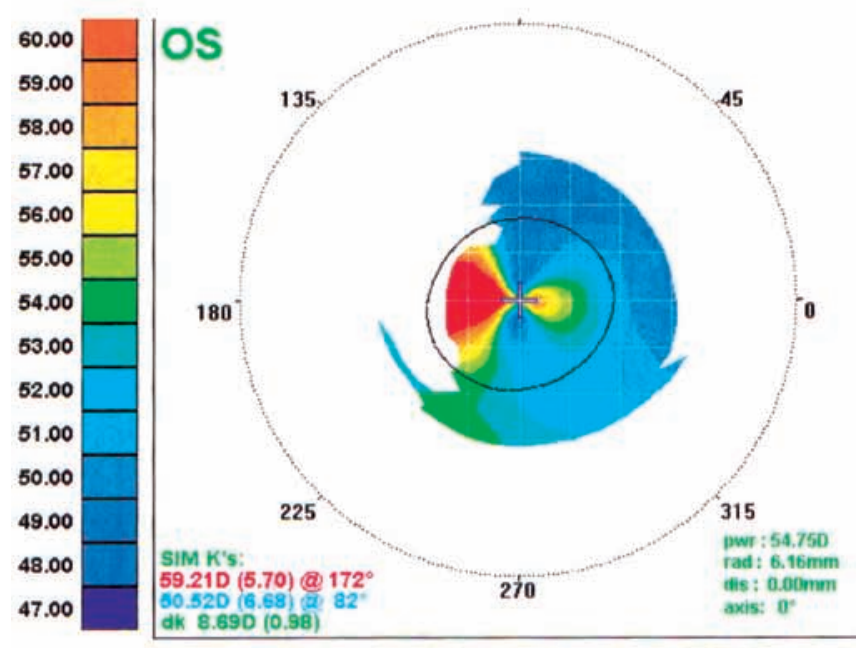

28 dias

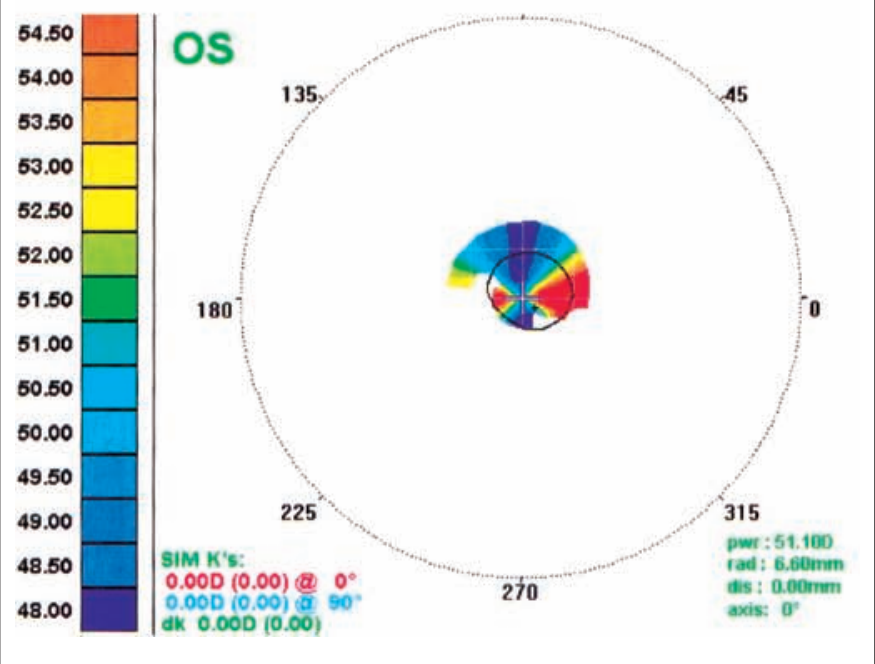

Figura 2 - Mudanças no mapa topográfico observadas em um animal do grupo experimento 2 , durante os períodos pré-operatório, pós-operatório de 14 dias e pós-operatório de 28 dias

\section{DISCUSSÃO}

Poucos são os estudos experimentais publicados envolvendo córnea de coelhos e anéis intra-estromais e, dentre eles, dois se destacam. Em 1987 alguns autores, realizaram o primeiro estudo experimental sobre o efeito do implante de anéis intra-estromais ("intrastromal corneal rings" - ICR) na córnea destes animais ${ }^{(1)}$. Acreditava-se que, por meio destes implantes, seria possível corrigir tanto erros miópicos quanto hipermetrópicos variando-se os diâmetros dos anéis. Dois animais foram utilizados neste estudo, nos quais foram implantados anéis intra-estromais na periferia corneana. Em um deles, ao se realizar aumento do diâmetro do anel, observou-se aplanamento central corneano, após 6 meses, demonstrado por ceratoscopia computadorizada. No outro, a diminuição do diâmetro do anel levou a aumento na curvatura corneana central, após esse período. Em 2000 alguns autores demonstraram, em olhos de coelhos, que a introdução de anéis intra-estromais com diferentes espessuras, porém com diâmetro constante, e a introdução de anéis com medidas constantes (diâmetro e espessura) em túneis de tamanhos diferentes geraram, no primeiro mês de estudo, aplanamento corneano central significativo ${ }^{(15)}$.

Estes dados não confirmaram o conceito inicial de constricção e aumento da curvatura corneana proposto anteriormente $^{(1)}$ e demonstraram que o efeito refratométrico do anel intra-estromal está relacionado, dentro de certos limites, a sua espessura. $\mathrm{Na}$ presente pesquisa, foram utilizados implantes com a mesma espessura e o mesmo diâmetro em todos os casos, pois já é sabido que quanto maior a espessura, maior o efeito obtido com o implante ${ }^{(3)}$.

Pesquisas realizadas anteriormente demonstraram que os resultados obtidos em olhos de coelhos foram significativos até o $14^{\circ}$ dia de pós-operatório e que, após 30 dias de cirurgia, as alterações corneanas induzidas pelo implante, comparadas ao grupo controle (não operado), não foram significativas, provavelmente devido às peculiaridades da córnea de coelho e às alterações histológicas induzidas pelo anel ${ }^{(15)}$. Embasados na literatura ${ }^{(15)}$ e sabendo do efeito temporário dos implantes em coelhos, os resultados desta pesquisa foram avaliados nos 14 primeiros dias após a cirurgia nos grupos 1 e 2, e após 14 dias da segunda cirurgia no grupo 2.

Técnicas de biomicroscopia, documentação fotográfica e ceratoscopia computadorizada normalmente utilizadas em seres humanos podem ser empregadas, sem necessidade de instrumentação especial, em pesquisa com coelhos. Limitações técnicas como dificuldade de posicionamento do animal durante o exame e distorções de imagem geradas por menisco lacrimal, entretanto, podem levar a grande variabilidade nos resultados de exames como a ceratoscopia computadorizada ${ }^{(1,16)}$.

Mesmo realizando-se os exames com todo o cuidado necessário e mesmo utilizando-se animais com peso e idade semelhantes, variações topográficas foram encontradas neste estudo. O implante do anel na periferia prejudicou a imagem da ceratoscopia computadorizada nesta região.

Dificuldades técnicas ao se realizar as cirurgias provavel- 
mente estavam relacionadas às diferenças anatômicas entre as córneas humana e de coelho. O fato de estas serem mais finas, mais curvas ${ }^{(15)}$ e mais elásticas ${ }^{(17)}$ gerou uma curva de aprendizado que demandou tempo do pesquisador para se adaptar às novas condições de cirurgia. $\mathrm{O}$ fato de o primeiro animal operado ter sofrido perfuração corneana durante o ato cirúrgico provavelmente está relacionado à espessura mais reduzida e maior elasticidade da córnea nestes animais.

Os resultados deste estudo confirmaram as já conhecidas biocompatibilidade e tolerância dos implantes de PMMA. Clinicamente, nos casos não complicados, a porção central da córnea manteve-se transparente durante o estudo e pequena opacidade corneana foi observada, em apenas 1 caso, no local da incisão, na periferia corneana. Os achados biomicroscópicos encontrados foram: hiperemia conjuntival (5 casos), descentração do anel ( 2 casos), opacidade corneana (1 caso) e infecção corneana (1 caso). Não ocorreram extrusão do anel, erosão corneana ou reação inflamatória de câmara anterior.

Poucos são os estudos experimentais disponíveis na literatura que analisam as complicações relacionadas ao implante de anel intra-estromal. Alguns autores encontraram como complicações mais freqüentes, em seu estudo envolvendo 96 coelhos, infecção corneana ( 8 olhos; $8,3 \%$ ) e neovascularização corneana ( 3 olhos; $3,1 \%$ ), ambas diretamente relacionadas ao grau de habilidade do cirurgião ${ }^{(15)}$. A hiperemia conjuntival encontrada em 5 coelhos deste estudo foi leve e transitória, durando aproximadamente três dias em todos os casos, e estava relacionada ao trauma cirúrgico. Apesar dos cuidados de assepsia e anti-sepsia tomados antes e durante o ato cirúrgico e dos colírios de antibiótico utilizados no pós-operatório, infecção cirúrgica é sempre uma complicação esperada, especialmente em se tratando de cirurgia em animais. Infecção corneana foi encontrada em apenas 1 coelho e optou-se por eliminá-lo do estudo.

Pesquisas envolvendo seres humanos também revelam grande variedade de complicações. Dentre estas, uma parcela significativa está associada, provavelmente, à curva de aprendizado do procedimento cirúrgico. Outros autores descreveram 8 casos de ceratite infecciosa, comprovados por exames laboratoriais, após implante de anel intra-estromal e enfatizaram necessidade de rápida intervenção nestes $\operatorname{casos}^{(18)}$. No estudo de 36 olhos realizado utilizando duas incisões para implante dos segmentos, as principais complicações encontradas foram: descentração do anel (1 olho), posicionamento assimétrico ( 2 olhos), profundidade inadequada ( 2 olhos), migração dos segmentos (2 olhos), extrusão dos anéis (5 olhos), conjuntivite ( 1 olho), ceratite bacteriana ( 1 olho) e hidropsia (1 olho $)^{(19)}$. Alguns autores observaram descentração (2 olhos), extrusão (10 olhos), ceratite bacteriana ( 1 olho) e ceratite disciforme $(1 \mathrm{olho})^{(20)}$.

No presente relato, a paquimetria pré-operatória média encontrada foi de $367,33 \pm 28,32$ micra para o olho direito e $355,16 \pm 20,83$ micra para o olho esquerdo. Tais medidas foram realizadas na média periferia corneana às 12 horas, no local onde seria realizada a incisão, e estão de acordo com o estudo realizado que encontraram espessura corneana central média de $356,11 \pm 14,34$ micra em olhos de coelhos ${ }^{(21)}$. Embora as medidas tenham sido realizadas em locais distintos da córnea nestes dois trabalhos, a comparação de resultados é possível devido à espessura corneana relativamente homogênea nestes animais, analisando-se do centro para a periferia ${ }^{(1)}$, o que contrasta com a córnea humana. Esta, ao contrário, apresenta espessamento do centro para a periferia.

No presente estudo, operou-se o olho esquerdo de todos os animais, mantendo-se o direito como controle. Objetivouse, assim, comparar o aplanamento corneano obtido com o implante dos anéis com aquele observado pelo crescimento dos animais. A curvatura da córnea do coelho sofre aplanamento durante seu crescimento ${ }^{(22)}$. Observa-se grande aplanamento, de até 6 dioptrias, entre 7 e 12 semanas de vida. Até a $20^{\mathrm{a}}$ semana de vida, a curvatura corneana do animal está em torno de 47 dioptrias. A análise realizada entre o peso e os valores ceratométricos, no pré-operatório, demonstrou que, no grupo de maior massa corpórea, os valores ceratométricos foram significativamente menores, concordando assim com a literatura. Os resultados da análise intragrupo demonstraram aplanamento corneano, obtido após o período de estudo (14 e 28 dias), em todos os quadrantes, nos animais do grupo controle 1 e 2, confirmando assim os dados obtidos na literatura.

Os resultados do grupo experimento 1 e 2 demonstraram aumento significativo dos valores ceratométricos nos setores nasal e temporal e diminuição desses nos setores superior e inferior. O setor superior foi o local onde se confeccionou a incisão. Verificou-se, $14^{\circ}$ dias após o período de estudo, aplanamento neste setor em todos os grupos; no entanto, nos grupos experimento 1 e 2, a diminuição dos valores foi mais evidente do que a ocorrida nos grupos controle 1 e 2 . Após 28 dias, houve novamente diminuição dos valores ceratométri$\cos$ no grupo 2, mais evidente que a ocorrida no grupo controle 2. É sabido que incisões arqueadas diminuem o astigmatismo e que incisões radiais aplainam a córnea no eixo da incisão. Sabe-se também que, quanto mais profundas as incisões, maior sua eficácia e que se perde parte do efeito refrativo após o processo de cicatrização. O local da incisão nesta pesquisa coincide com o local entre os ápices dos dois segmentos de implante; por isso, não se pode concluir que a incisão teria algum efeito sobre o aplanamento da córnea, pois o mesmo efeito foi observado no setor inferior. Talvez o aplanamento ocorra no eixo onde estão posicionadas as extremidades dos segmentos implantados e não necessariamente onde se encontra a incisão.

Nos grupos experimento 1 e 2 foi observado aumento significativo da curvatura corneana nos setores nasal e temporal, locais onde se localizava o corpo do anel. No grupo experimento 2 , na primeira fase do estudo, o implante de apenas um segmento induziu a encurvamento maior no setor nasal, que recebeu o implante, gerando assim astigmatismo assimétrico. Nos grupos controle 1 e 2, foi observado aplanamento corneano significativo nestes dois setores. Outros autores descreveram uma modificação no implante do INTACS no tratamento do ceratoco- 
ne ${ }^{(14)}$. Foram implantados segmentos assimétricos em 74 olhos de 50 pacientes com ceratocone. O segmento mais espesso foi implantado inferiormente e o segmento mais fino, superiormente. Concluíram nesta pesquisa que implantes assimétricos podem melhorar a acuidade visual e reduzir as distorções provocadas pelo astigmatismo irregular.

$\mathrm{O}$ anel corneano intra-estromal tem sido indicado basicamente nos casos de ectasia corneana caracterizados por protrusão e afinamento localizados. Nesta pesquisa, foram utilizadas córneas de coelhos, sem alterações topográficas significativas, que apresentavam características distintas das observadas na córnea humana (esfericidade, maior elasticidade, maior curvatura e menor espessura). Os resultados deste estudo não podem ser extrapolados para o ser humano, no entanto, eles foram muito evidentes e demonstraram claramente as alterações que ocorrem na córnea em decorrência de um implante corneano intra-estromal.

Mais pesquisas devem ser incentivadas com objetivo de esclarecer controvérsias, ainda existentes, a respeito de posicionamento de incisão e indicação de implantes assimétricos.

\section{CONCLUSÕES}

Os efeitos do implante de anel corneano intra-estromal sobre a curvatura corneana foram:

1. Aplanamento no eixo onde se localizam as extremidades dos segmentos.

2. Aumento significativo da curvatura no setor onde se localiza o corpo do segmento.

3. Astigmatismo assimétrico após implante de apenas um segmento.

\section{ABSTRACT}

Purpose: To evaluate the effect of intrastromal corneal ring implantation on corneal curvature in rabbits. Methods: Thirty eyes of 15 rabbits were divided into two groups: group 1 with 7 animals and group 2 with 8 animals. The left eye underwent surgery and the right eye was used as control. Ultrasound pachymetry and corneal topography were performed prior to surgery. Anterior segment biomicroscopy and corneal topography were performed after surgery. In group 1, implantation of two ring segments was performed. In group 2, ring segments were implanted separately. Topographical findings, before and after surgery, were compared through quantitative and qualitative alterations and divided into four quadrants. Results: Quantitative analysis showed no significant difference between the groups in the preoperative period. Experiment group 1 showed nasal $(\mathrm{p}=0.02)$ and temporal $(\mathrm{p}=0.04)$ corneal steepening, compared to control group 1. Experiment group 2 showed nasal and temporal corneal steepening and superior and inferior corneal flattening ( $\mathrm{p}=0.02$ for all quadrants) compared to the control group 2. Qualitative analysis showed uniform astigmatism in both control groups. In experi- ment group 1, against-the-rule regular symmetrical astigmatism was observed after surgery. In experiment group 2, againstthe-rule regular asymmetrical astigmatism was found after implantation of the first ring segment, and against the rule regular symmetrical astigmatism was observed after implantation of the second segment. Conclusion: In this study, significant corneal flattening occurred in quadrants where ring segments extremities were located and corneal steepening was observed in quadrants where its body was located. Implantation of one ring segment induces asymmetrical astigmatism.

Keywords: Corneal topography; Prosthesis implantation; Corneal stroma/surgery; Astigmatism; Rabbits

\section{REFERÊNCIAS}

1. Fleming JF, Reynolds AE, Kilmer L, Burris TE, Abbot RL, Schanzlin DJ. The intrastromal corneal ring: two cases in rabbits. J Refract Surg. 1987;3(6): 227-32.

2. Burris TE, Ayer CT, Evensen DA, Davenport JM. Effects of intrastromal corneal ring size and thickness on corneal flattening in human eyes. Refract Corneal Surg. 1991;7(1):46-50.

3. Burris TE, Baker PC, Ayer CT, Loomas BE, Mathis ML, Silvestrini TA. Flattening of central corneal curvature with intrastromal corneal rings of increasing thickness: an eye-bank eye study. J Cataract Refract Surg. 1993;19 Suppl:182-7.

4. Assil KK, Barret AM, Fouraker BD, Schanzlin DJ. One-year results of the intrastromal corneal ring in nonfunctional human eyes. Arch. Ophthalmol. 1995;113(2):159-67.

5. Schanzlin DJ, Asbell PA, Burris TE, Durrie DS. The intrastromal corneal ring segments; phase II results for the correction of myopia. Ophthalmology. 1997; 104(7):1067-78.

6. Twa MD, Karpecki PM, King BJ, Linn SH, Durrie DS, Schanzlin DJ. One-year results from the phase III investigation of the KeraVision Intacs ${ }^{\mathrm{TM}}$. J Am Optom Assoc. 1999;70(8):515-24.

7. Colin J, Cochener B, Savary G, Malet F. Correcting keratoconus with intracorneal rings. J Cataract Refract Surg. 2000;26(8):1117-22. Comment in: J Cataract Refract Surg. 2000;26(8):1099-100. J Cataract Refract Surg. 2001;27 (3):341.

8. Colin J, Cochener B, Savary G, Malet F, Holmes-Higgin D. Intacs inserts for treating keratoconus. Ophthalmology. 2001;108(8):1409-14.

9. Moreira H, Oliveira CS, Godoy G de, Wahab SA. Anel intracorneano de Ferrara em ceratocone. Arq Bras Oftalmol. 2002;65(1):59-63.

10. Siganos D, Ferrara P, Chatzinikolas K, Bessis N, Papastergiou G. Ferrara intrastromal corneal rings for the correction of keratoconus. J Cataract Refract Surg. 2002;28(11):1947-51

11. Siganos CS, Kymionis GD, Kartakis N, Theodorakis MA, Astyrakakis N, Pallikaris IG. Management of keratoconus with Intacs. Am J Ophthalmol. 2003;135(1):64-70.

12. Cunha PFA, Alves EAF, Silva FBD, Cunha GHA. Estudo das modificações oculares induzidas pelo implante estromal do anel de Ferrara em portadores de ceratocone. Arq Bras Oftalmol. 2003;66(4):417-22.

13. Oliveira CS, Moreira H, Wahab SA, Godoy G. Análise de nova técnica para o implante do anel de Ferrara no ceratocone. Arq Bras Oftalmol. 2004;67(3):509-17.

14. Boxer Wachler BS, Christie JP, Chandra NS, Chou B, Korn T, Nepomuceno R. Intacs for keratoconus. Ophthalmology. 2003;110(5):1031-40. Erratum in: Ophthalmology. 2003;110(8):1475.

15. Hartmann C, Rieck PW, Holzkamper C, Pouliquen Y, Renard G. The intrastromal corneal ring in clinical refractive surgery: reference to results in rabbit eyes. Graefes Arch Clin Exp Ophthalmol. 2000;238(6):465-71.

16. Goins KM, Karp KO, Gabrielian K. Minoxidil-induced alteration of corneal topography after radial keratotomy. J Cataract Refract Surg. 1998;24(5):612-8.

17. Hoeltzel DA, Altman P, Buzard K, Choe K. Strip extensiometry for comparison of the mechanical response of bovine, rabbit, and human corneas. $\mathrm{J}$ Biomech Eng. 1992;114(2):202-15.

18. Hofling-Lima AL, Branco BC, Romano AC, Campos MQ, Moreira H, Miranda $\mathrm{D}$, et al. Corneal infections after implantation of intracorneal ring segments. Cornea. 2004;23(6):547-9. Erratum in: Cornea. 2004;23(7):table of contents. Casanova, Fabio [corrected to Casanova, Fabio H].

19. Miranda D, Sartori M, Francesconi C, Allemann N, Ferrara P, Campos M. 
Ferrara intrastromal corneal ring segments for severe keratoconus. J Refract Surg. 2003;19(6):645-53.

20. Kwitko S, Severo NS. Ferrara intracorneal ring segments for keratoconus. J Cataract Refract Surg. 2004;30(4):812-20.

21. Schulz D, Iliev ME, Frueh BE, Goldblum D. In vivo pachymetry in normal eyes of rats, mice and rabbits with the optical low coherence reflectometer. Vision Res. 2003;43(6):723-8.

22. Bryant MR, Kampmeier J, Er H, Kasetsuwan N, Sanchez-DiMartino D, Shah SS, McDonnell PJ. PRK-induced anisometropia in the rabbit as a model of myopia. Graefes Arch Clin Exp Ophthalmol. 1999;237(2):161-5.

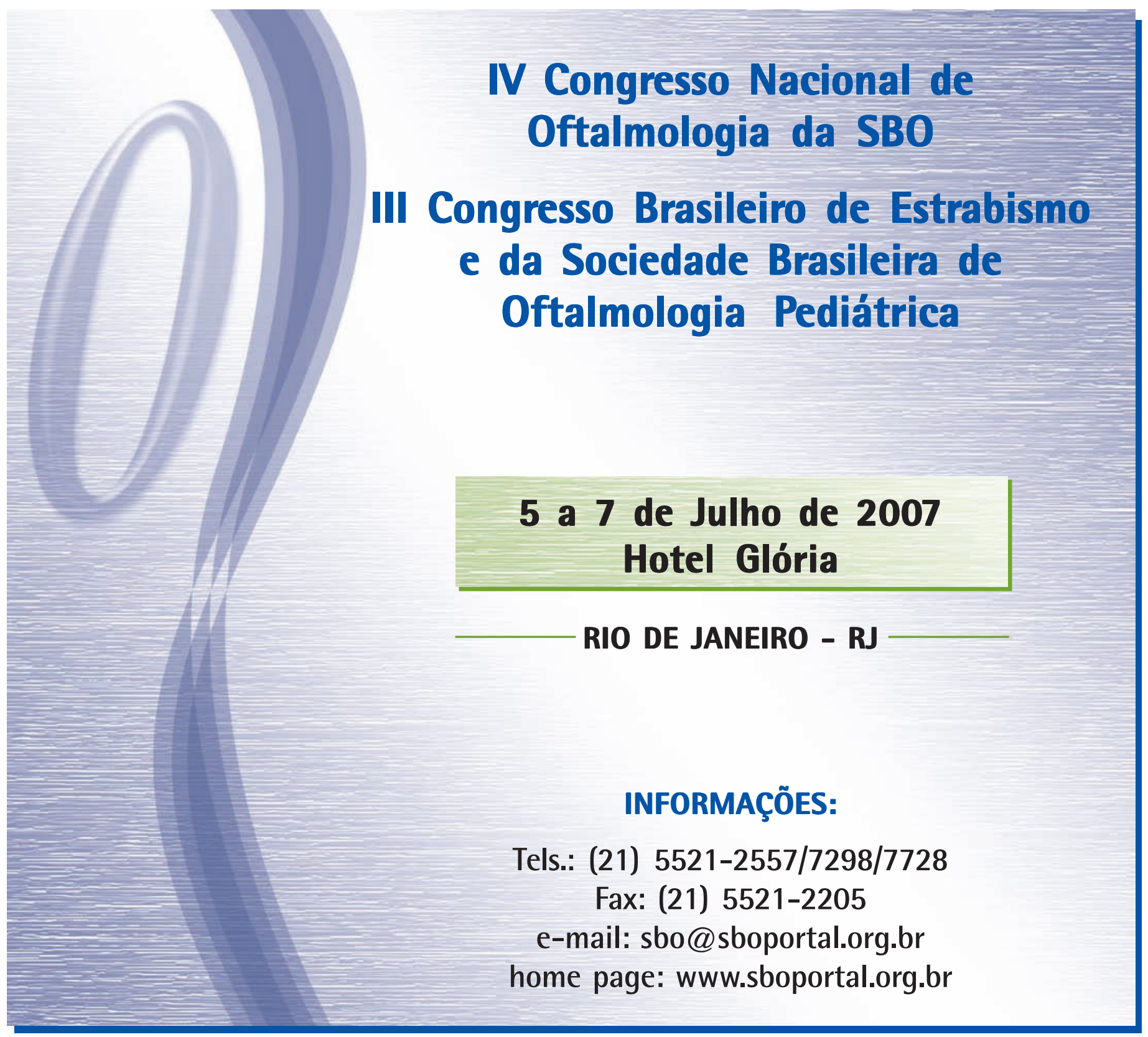

